\title{
Reflectance anisotropy spectroscopy assessment of the MOVPE nucleation of GaInP on germanium ( 100$)$
}

\author{
E. Barrigon ${ }^{\mathrm{a}, *}$, B. Galiana ${ }^{\mathrm{b}}$, I. Rey-Stolle $^{\mathrm{a}}$ \\ ${ }^{a}$ Instituto de Energía Solar, Universidad Politécnica de Madrid ETSI de Telecomunicación, Avda. Complutense 30, 28040 Madrid, Spain \\ b ICMM-CSIC Sor Juana Ines de la Cruz 3, 28049 Madrid, Spain
}

Keywords:

A1. Reflectance anisotropy spectroscopy

A1. Growth monitoring

A2. Metalorganic vapor phase epitaxy

B1. Phosphides

B2. Semiconducting germanium

\begin{abstract}
A B S T R A C T
This work summarizes the observations made on the variation and time evolution of the reflectance anisotropy signal during the MOVPE growth of GaInP nucleation layers on Germanium substrates. This in situ monitoring tool is used to assess the impact of different nucleation routines and reactor conditions on the quality of the layers grown. This comparison is carried out by establishing a correlation between reflectance anisotropy signature at $2.1 \mathrm{eV}$ and the morphology of the epilayers evaluated by atomic force microscopy (AFM). This paper outlines the potential of reflectance anisotropy to predict, explore, and therefore optimize, the best growth conditions that lead to a high quality III-V epilayer on a Ge substrate.
\end{abstract}

\section{Introduction}

During the last two decades there has been an increasing interest in the epitaxial growth of III-V compounds on germanium (Ge). Today germanium wafers are mostly used in the manufacture of solar cells (both for space and terrestrial concentrator systems) though there are other emerging applications such as photodetectors, LEDs, CMOS and even HEMTs [1].

The growth of III-V polar semiconductor on a non-polar Ge wafer is not a straightforward task [2]. On the one hand, a high quality template for further III-V epitaxial growth has to be created, with an electrically neutral heterointerface, and no anti-phase domains (APD), misfit dislocations, hillocks or other kinds of 3D defects $[3,4]$ that affect the morphology of the epilayers. On the other hand, the diffusion processes that take place across the heterointerface have to be controlled. In summary a good heteronucleation routine has to control all these issues and, in addition, cope with the variability that is sometimes observed in the quality of Ge substrates [5].

To achieve these goals, it is of key interest to be able to monitor the surface processes during the nucleation process. Reflectance Anisotropy Spectroscopy (RAS) is an excellent tool for this purpose in an MOVPE environment where other techniques are not usable due to the lack of ultra-high vacuum. Essentially RAS measures the difference in reflectance of normal incidence polarized light between two orthogonal crystal directions in the surface plane, normalized to the mean reflectance. Excellent reviews of the foundations and possible applications in epitaxial growth of this technique can be found elsewhere [6-8]. The basic knowledge of the state of the surface at an atomic level, i.e. the surface reconstruction, of the Ge wafer during the pre-growth moments and the first monolayers of the III-V compound grown on it can be advantageous in order to be able to circumvent and/or understand some of the aforementioned problems [7,9].

In this paper we present the analysis of the RAS signal for the growth of GaInP nucleation layers on Ge, during the time prior to the growth (i.e. pre-nucleation) and during the first stages of the epitaxial process (i.e. nucleation), for a variable set of reactor situations and MOVPE growth conditions. The use of GaInP nucleation layers on $\mathrm{Ge}$ presents some advantages as compared to the use of GaAs, particularly for our case of solar cell applications ( $P$ possesses a lower diffusion length into Ge than As, which allows the formation of shallower emitters in the bottom subcell) [10]. However, there are very few reports in the literature dealing with RAS of Ge, regarding the optimization of a device. Only Olson [9] reported some RAS spectra of $\mathrm{Ge}(100)$ under $\mathrm{AsH}_{3}$ and under $\mathrm{H}_{2}$, in a clean and in a coated MOVPE reactor. Nevertheless, some surface science publications about different Ge orientations [11-14] can be found.

Our goal in analyzing the RA signal is to gain insight into the nucleation process and thus find an optimized MOVPE nucleation routine. In parallel, the RAS signal can be used to control the reproducibility of the surface reconstructions of the $\mathrm{Ge}$ wafers prior to growth, and thus to assure the quality of the wafer and/or the state of the reactor, which may impact the achievement of a successful heteroepitaxy.

\section{Experimental}

Samples in this study were grown by MOVPE in a horizontal, low pressure, AIX200/4 reactor. The precursors used were 


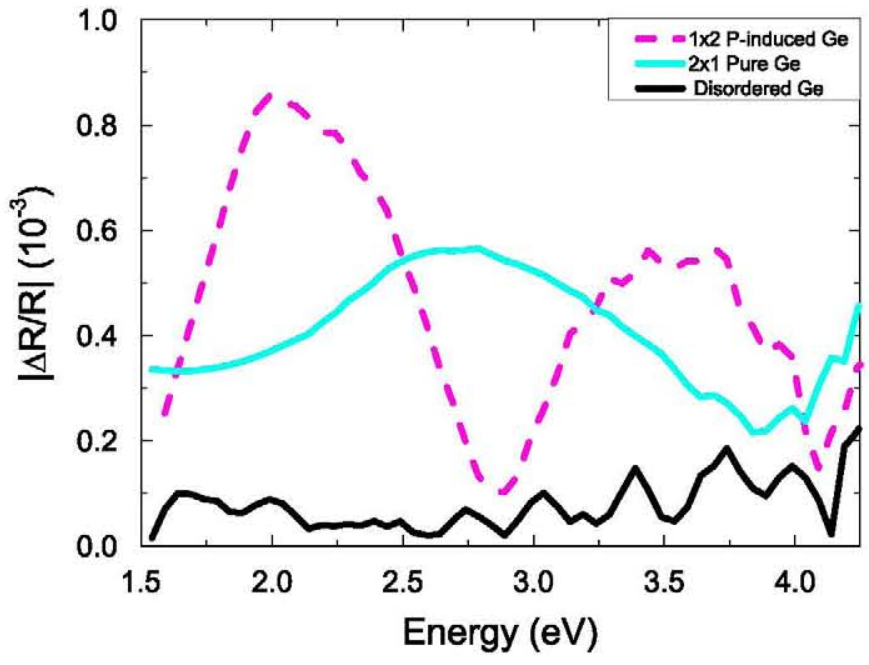

Fig. 1. RAS spectra of pure, P-induced and disordered Ge.

trimethylgallium (TMGa), trimethylindium (TMIn), pure phosphine $\mathrm{PH}_{3}$ and pure arsine $\left(\mathrm{AsH}_{3}\right)$. The growths were carried out using 14 slpm of Pd-purified hydrogen as the carrier gas at a total reactor pressure of 100 mbar.

The Ge substrates used share the same main characteristics, being oriented $(100)$ with a miscut of $6^{\circ}$ towards the nearest (111) plane, doped with gallium (p-type) up to a resistivity of $1.3 \times 10^{-3} \Omega \mathrm{cm}$. The growth does not take place on full wafers but on pieces of around $2 \times 2 \mathrm{~cm}^{2}$. Thus, wafers are unpacked from their individual boxes in a laminar flow hood, which provides locally class 10 conditions. No ex situ cleaning was performed on the pieces.

The heteronucleation of GaInP on Ge consists of two main parts. The first one is the so called pre-nucleation, which is made in order to clean and prepare the wafer. This is done by a high temperature heat bake $\left(650^{\circ} \mathrm{C}\right)$ followed by a $\mathrm{PH}_{3}$ anneal which was varied in terms of time and partial pressure of $\mathrm{PH}_{3}$. The second one is the nucleation of the GaInP layer itself, $70 \mathrm{~nm}$ grown at $0.26 \mathrm{~nm} / \mathrm{s}$, at $600^{\circ} \mathrm{C}$ and with a $\mathrm{V} / \mathrm{III}$ ratio of 420 . Finally, this layer is capped with a protective layer of GaInAs of around $400 \mathrm{~nm}$.

The RAS spectrometer is a commercial tool (EpiRAS 2000) with real temperature measurement of the wafer by pyrometry at $950 \mathrm{~nm}$. All the temperatures mentioned were obtained by this procedure. The RAS measurements presented throughout this work are taken in transient mode at $2.1 \mathrm{eV}$, and are presented as absolute values, due to the rotation of the wafers for growth uniformity purposes. This energy was chosen due to several factors. Firstly, as can be seen in Fig. $1,2.1 \mathrm{eV}$ is a maximum for the $1 \times 2$ surface reconstruction (one of the critical points of bulk $\mathrm{Ge}$ ), and recording the $2.1 \mathrm{eV}$ signal therefore allows detecting changes in the surface reconstruction. Furthermore, the reflectivity at this point is high enough, leading to a high RAS signal to noise ratio.

The surface morphology of the GaInP layers was examined by AFM using a Digital Instruments-Multimode IIla microscope working in tapping mode, and images were processed using a free software [15]. Conventional $\mathrm{Si}$ cantilevers with a typical resonance frequency of $300 \mathrm{kHz}$ were used as tips. To analyze the morphology of the GaInP nucleation layer, the GaInAs cap was selectively etched using $\mathrm{NH}_{4} \mathrm{OH}: \mathrm{H}_{2} \mathrm{O}_{2}: \mathrm{H}_{2} \mathrm{O}(2: 1: 10)$.

\section{Results}

The main experiments developed for this study are summarized in Table 1. They can be divided into two main categories, depending on the control (or lack of control) of the desorption of
Table 1

Brief summary of the different samples analyzed in this work and their RMS roughness as measured by AFM.

\begin{tabular}{llll}
\hline Case & $\begin{array}{l}\text { State of the } \\
\text { reactor }\end{array}$ & PH3 anneal & RMS (nm) \\
\hline A & Coated & None & 1.20 \\
B & Coated & $60 \mathrm{~s}, 0.8 \mathrm{mbar}$ & 0.84 \\
C & Coated & $60 \mathrm{~s}, 2.8 \mathrm{mbar}$ & 0.58 \\
D & After high T bake & None & 0.519 \\
E & Uncoated & None & 0.643 \\
F & Coated & PH & 0.589 \\
& & complete run & \\
\hline
\end{tabular}

species from the hot reactor walls and susceptor during the heatup of the sample and during the initial anneal at high temperature. The following subsections describe in detail both sets of experiments.

\subsection{Experiments without control of the desorption of deposited species in the reactor}

The first batch of samples $(A, B, C)$ were grown in a reactor in which III-V compounds are routinely grown (i.e. a reactor coated with III-V arsenides and phosphides) where nothing was done to prevent the impact of desorption of species on the growth. This means that during the heat-up step of the sample and during the initial anneal at high temperature there is a low, though uncontrolled, overpressure of group $\mathrm{V}$ and group III species desorbing from the susceptor and walls of the reactor. The heatup cycle and the high temperature anneal being identical, the main difference between the samples involved in these experiments ( $A$ to $\mathrm{C}$ in Table 1 ) is the partial pressure of $\mathrm{PH}_{3}$ used in the hydride anneal just before the actual growth starts.

In Fig. 2 the absolute value of the reflectance anisotropy (RA) signal at $2.1 \mathrm{eV}$ is presented for the last minutes of the pre-nucleation treatment and the whole growth of the GaInP nucleation layer (the part of the signal associated with the growth of the GaInAs cap layer is omitted to magnify the time slot relevant for this study). In the lower part of the figure, the partial pressure of $\mathrm{PH}_{3}$ during the growth is also shown. For the three samples, the starting point for the RA signal of the Ge wafer is practically zero.

For the three cases, when $\mathrm{PH}_{3}$ is opened, a spike in the RA signal is observed. For sample A this spike occurs just when the GaInP growth starts, while for samples $B$ and $C$ it takes place $60 \mathrm{~s}$ before, as the anneal under $\mathrm{PH}_{3}$ starts. Apart from the location of the spike, this anneal does not change the RA signal, as can be seen by comparing the trace of sample $B$ (annealed under a low pressure of $\mathrm{PH}_{3}$ ) to that of sample $\mathrm{A}$ (no anneal under $\mathrm{PH}_{3}$ ). In Fig. 1 is shown the spectrum of typical disordered Ge surface obtained in the two situations. This indicates that annealing under low flows of $\mathrm{PH}_{3}$ does not seem to affect significantly the state of the surface of the germanium wafers. In this case, it is mostly induced by the uncontrolled desorption of species from the walls of the reactor and susceptor. On the other hand, for sample $\mathrm{C}$ annealed under a high flow of $\mathrm{PH}_{3}$, a sudden increase in the level of the RA signal is measured when $\mathrm{PH}_{3}$ starts being injected into the reactor. Regular spectroscopic analyses performed under the same partial pressure of $\mathrm{PH}_{3}$ as sample $\mathrm{C}$ and in a wide range of temperatures $\left(580-650^{\circ} \mathrm{C}\right)$, indicate that this change corresponds to a $1 \times 2$ surface reconstruction (see Fig. 1) induced by $\mathrm{P}$ adatoms, in agreement with the observations of other authors $[9,16]$. 


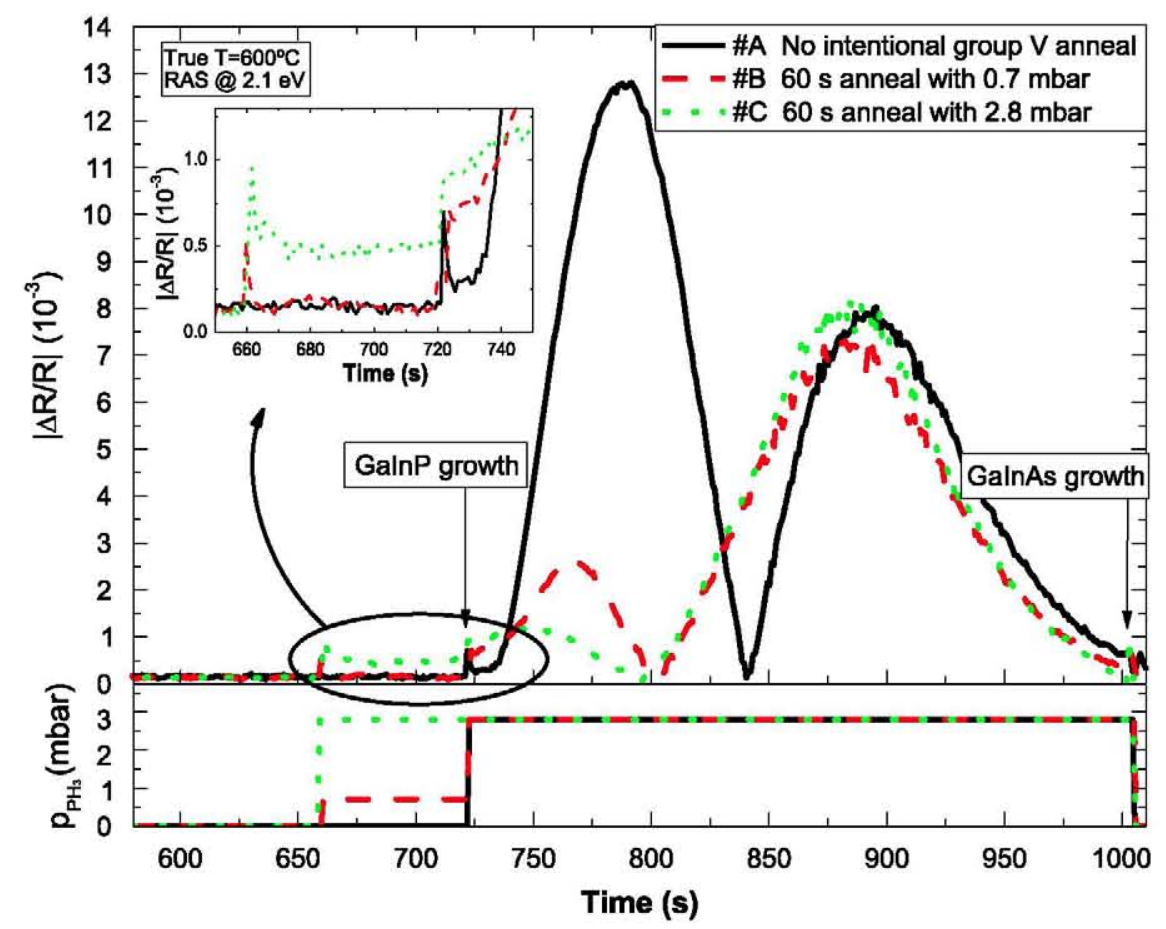

Fig. 2. Time resolved RA signal at $2.1 \mathrm{eV}$ and $600^{\circ} \mathrm{C}$ for samples $\mathrm{A}, \mathrm{B}$ and $\mathrm{C}$ of the last stage of pre-nucleation and the GalnP nucleation layer.

The moment when GalnP growth actually starts (i.e. the moment when TMGa and TMIn are injected) is marked in Fig. 2 with a vertical arrow. At this stage, the signal starts to oscillate, as the reflectance does (not shown here) due to Fabry-Perot interferences. The main difference observed between samples $A$ to $C$ is the magnitude and duration of the first oscillation of the RA signal. The peaks of the first oscillation of the RA signal due to the growth of GalnP are $13 \times 10^{-3}, 2.7 \times 10^{-3}$ and $1.2 \times 10^{-3}$ for cases A, B and C, respectively. The peak of the second oscillation is virtually identical for the three cases.

In Fig. 3 the $3 \times 3 \mu \mathrm{m}$ AFM scans of the GalnP layer of these three samples are shown. For each case, the image on the left shows the topography (all with the same scale), and the one on the right the amplitude (to facilitate the interpretation). The RMS roughness values on $A, B$ and $C$ are $1.20 \mathrm{~nm}, 0.84 \mathrm{~nm}, 0.58 \mathrm{~nm}$, respectively. Some evidence of step bunching is also noticeable in the AFM images of samples A and B.

\subsection{Experiments minimizing the effects of the desorption of deposited species in the reactor}

The goal of this second batch of experiments (samples D, E, F) is to analyze, with a fixed pre-nucleation condition (no PH3 anneal), whether the state of the reactor may affect the quality of the epilayers and if RAS can be used to monitor such differences.

Sample D was grown after performing a long high temperature bake of the reactor, with a temperature significantly higher than the nominal growth temperature, in order to desorb impurities from the susceptor and walls of the reactor. Before growth of sample E, the reactor was cleaned, i.e. the susceptor, liner and holders were changed to uncoated fresh new ones. Sample F was grown in a coated reactor but under a partial pressure of $\mathrm{PH}_{3}$ from the beginning of the heat-up. The idea behind all these experiments was to eliminate the influence of the uncontrolled desorption of group-V and/or group-III species from hot reactor parts. In cases $\mathrm{D}$ and $\mathrm{E}$ this elimination occurs by avoiding the desorption process while in case $\mathrm{F}$ it takes place by making the desorption process irrelevant by adding a controlled and much larger amount of the group- $V$ element.

In Fig. 4 the RAS signals for these three cases (D, E, F) are compared to that for sample $C$ from last section. Despite having used the same pre-nucleation treatment as for sample A (i.e. lack of hydride during the anneal prior to growth start), the RA signal for sample D is very similar to that of case C. Also, the RA signals of samples $\mathrm{E}$ and $\mathrm{F}$ during the growth of the GalnP nucleation layer are very similar to those for samples $C$ and $D$.

However, there are some differences between these samples' RA signals before TMGa and TMIn are injected. As can be seen in the inset of Fig. 4, the RA signal of sample $E$ is not zero from the beginning, but has a level of $0.4 \times 10^{-3}$. In this case, an RA spectrum recorded at this point (see Fig. 1) suggests that this value corresponds to a pure Ge $2 \times 1$ surface reconstruction. For sample $F$, the RA signal is also higher than zero before GaInP growth starts, however, the RA spectrum indicates a $1 \times 2$ surface reconstruction (see example in Fig. 1) as a result of the presence of $\mathrm{PH}_{3}$, as occurred for sample $\mathrm{C}$.

The AFM scans of samples D to F are similar to that of sample $C$, and are therefore not included. The RMS roughness is $0.519 \mathrm{~nm}$, $0.643 \mathrm{~nm}, 0.589 \mathrm{~nm}$ for cases D, E, F, respectively.

\section{Discussion}

For the experiments carried out without control of the desorption of deposited species in the reactor, a clear correlation between the first oscillation of the RA signature at $2.1 \mathrm{eV}$ and the epilayer morphology could be established: the higher the anisotropy during the first oscillation of the GaInP nucleation layer, the higher the roughness of the layer, as seen in other systems like $n$ GaInP-n AlGaInP [17]. These differences in roughness could be the product of different nucleation modes, i.e. basically island formation, or other perturbations of the ideal 2D step-flow growth mode as observed on the AFM scans. In this 


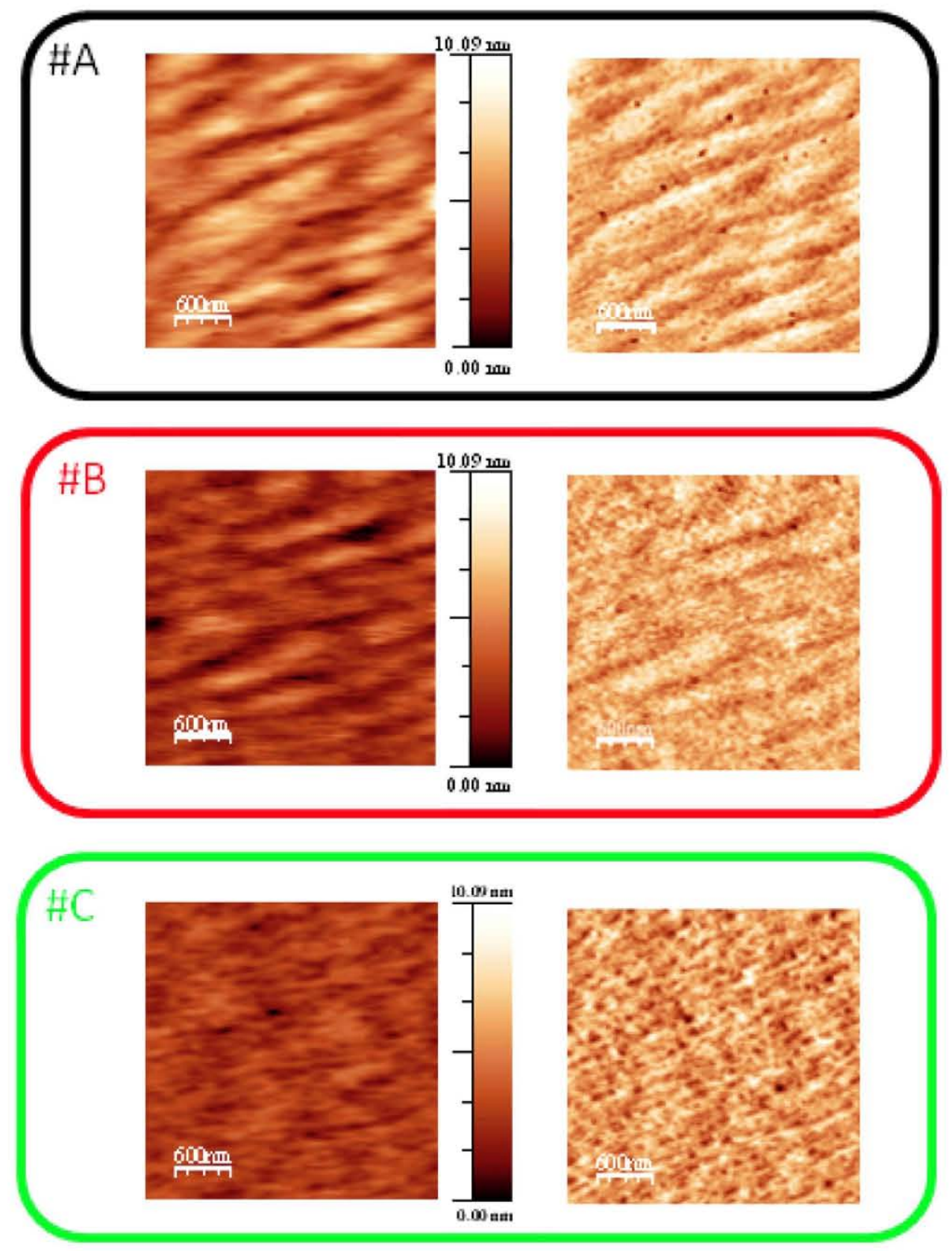

Fig. 3. AFM scans of samples A, B and C.

set of experiments, only when the RA signal on the wafer prior to growth is not zero does the epilayer obtained present an acceptable RMS roughness. This means that the formation of a dominant domain with $1 \times 2$ surface configuration (resulting from a sufficient $\mathrm{PH}_{3}$ anneal prior to growth), leads to a good morphology for the epilayer grown. The key point here is that RA allows to ascertain the importance of having a single domain surface prior to growth, as indicated by empirical evidence [18].

On the other hand, for the experiments carried out with a controlled pre-nucleation atmosphere (either pure $\mathrm{H} 2$ or $\mathrm{H} 2$ with a controlled phosphine overpressure), the RA signature associated with the low RMS roughness is always obtained. In other words, in samples D and E we obtain the type of RA that is related to a reasonably good morphology, although those samples were not heated up prior to growth under high $\mathrm{PH} 3$ partial pressure as was the case for sample $\mathrm{C}$. This fact seems to indicate that the key factor to attain good morphology is the presence of single-domain surface -either $1 \times 2$ or $2 \times 1$; either pure Ge or P-induced, which is in total agreement with the evidence presented in the literature for other heteroepitaxial systems.

Another interesting consequence of the comparison of both sets of experiments is that the processes that spoil the morphology of sample A and, to a lesser extent, of sample B are related to the uncontrolled desorption of species from reactor walls and susceptor during the pre-nucleation treatment. The nature of this process is not well understood yet and will be the subject of future investigations.

\section{Conclusions}

RAS is a powerful tool for monitoring and optimizing the growth of GaInP on Ge in a MOVPE reactor. This in situ tool has been applied to the analysis of different nucleation routines and 


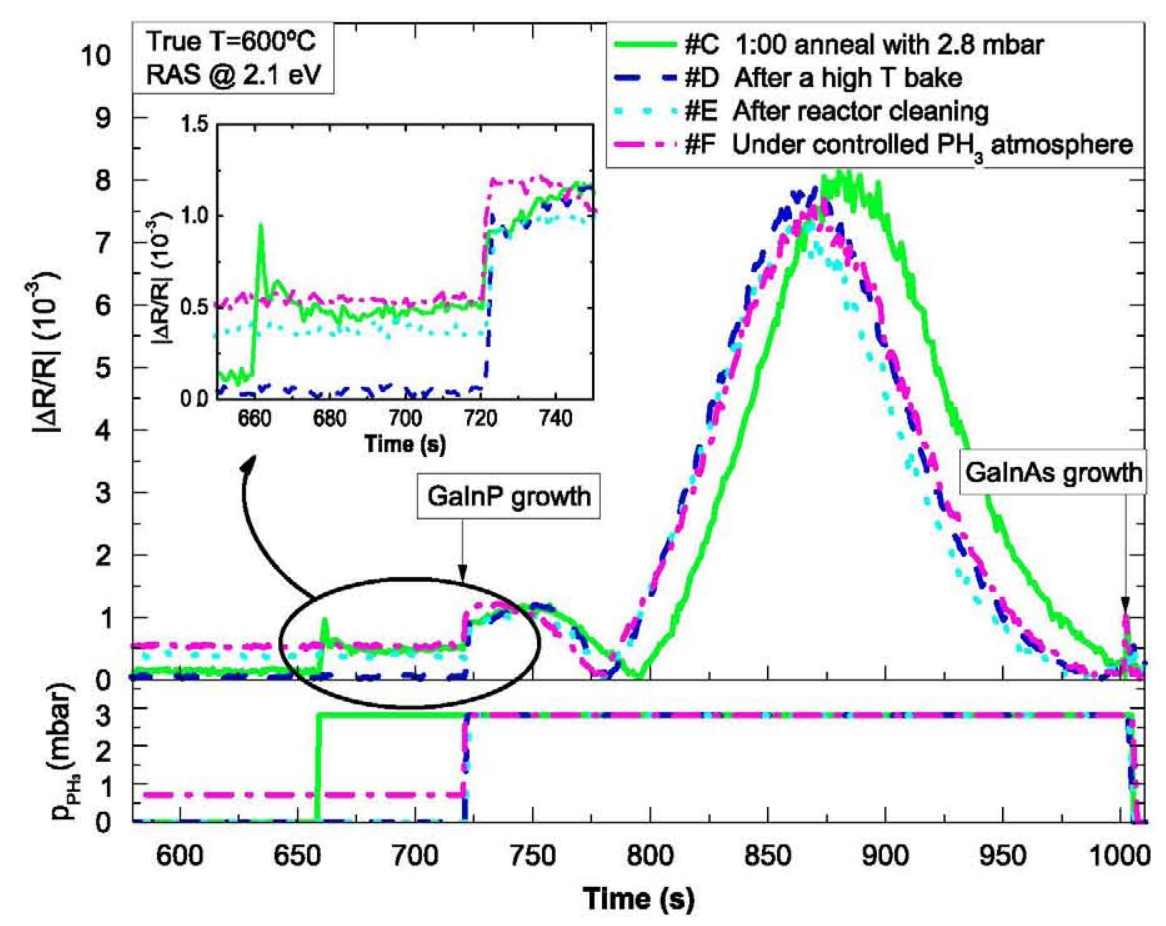

Fig. 4. Time resolved RA signal at $2.1 \mathrm{eV}$ and $600^{\circ} \mathrm{C}$ for samples C, D, E and F of the last stage of pre-nucleation and the GaInP nucleation layer.

reactor conditions and their effects on the morphology of the layers grown. It has been shown that the RA signal at $2.1 \mathrm{eV}$ can be observed to guide the growth of a nucleation layer with minimum roughness. The key feature of this signature is the height of the peak reached by the first oscillation, which is directly related to the RMS roughness of the GaInP epilayer (i.e. the higher the peak the larger the RMS roughness), as measured by AFM.

One of the main results of the study is that nucleation layers with good morphology can be obtained if the Ge surface reconstruction is either mostly $(1 \times 2)$ or $(2 \times 1)$. This can be accomplished by using a variety of approaches such as (a) eliminating the presence of contamination (i.e. using a clean reactor); (b) depleting the reactor of volatile contaminants with a high temperature bake before growth; (c) minimizing the role of desorbed species by maintaining a sufficient overpressure of phosphine from the start of the process; or (d) restoring the surface with an intense $\mathrm{PH}_{3}$ flush just prior to the growth of the nucleation layer. In all these cases the RA signature at $2.1 \mathrm{eV}$ during GaInP growth is very similar and the RMS roughness of the GaInP layers grown on $\mathrm{Ge}$ is similar and low $(0.5 \mathrm{~nm})$. On the other hand, in other situations where the Ge surface prior to growth is controlled by species having desorbed from the susceptor and reactor walls, the RA signal evolution during GaInP growth presents evident changes with respect to the optimum situation. Therefore, it has been demonstrated that RAS can guide both the understanding of the Ge surface before the growth starts and subsequent assessment and optimization of a heteronucleation routine for GalnP on $\mathrm{Ge}$.

\section{Acknowledgements}

Part of the funding for this research was provided by the Spanish Ministry of Education and Science (Ministerio de Educación y Ciencia) through Projects GENESIS-FV (CSD2006-004), TEC200801226 and TEC2009-11143. The Regional Government of Madrid (Comunidad Autónoma de Madrid) also contributed under Contract
S- 505/ENE/0310 (NUMANCIA Program). Enrique Barrigon holds a Ph.D. scholarship of the Spanish Ministry of Science (FPI programm). The authors are deeply indebted to J. Bautista for his continuous technical support.

\section{References}

[1] B. Depuydt, A. Theuwis, l. Romandic, Germanium: from the first application of Czochralski crystal growth to large diameter dislocation-free wafers, Mater. Sci. Semicond. Process. 9 (2006) 437-443.

[2] Y. Li, G. Salviati, M.M.G. Bongers, L. Lazzarini, L. Nasi, L.J. Giling, On the formation of antiphase domains in the system of GaAs on Ge, J. Cryst. Growth 163 (1996) 195-202.

[3] B. Galiana, E. Barrigon, I. Rey-Stolle, V. Corregidor, P. Espinet, C. Algora, E. Alves, Compositional analysis and evolution of defects formed on GalnP epilayers grown on Germanium, Superlattices Microstruct. 45 (2009) 277-284.

[4] A. Lankinen, L. Knuuttila, P. Kostamo, T. Tuomi, H. Lipsanen, P. McNally, L. O'Reilly, Synchrotron topography and X-ray diffraction study of GalnP layers grown on GaAs/Ge, J. Cryst. Growth 311 (2009) 4619-4627.

[5] I. Rey-Stolle, E. Barrigón, B. Galiana, C. Algora, Analysis of germanium epiready wafers for III-V heteroepitaxy, J. Cryst. Growth 310 (2008) 4803-4807.

[6] P. Weightman, D.S. Martin, R.J. Cole, T. Farrell, Reflection anisotropy spectroscopy, Rep. Prog. Phys. 68 (2005) 1251

[7] W. Richter, In-situ observation of MOVPE epitaxial growth, Appl. Phys. A 75 (2002) 129-140

[8] J.-T. Zettler, Characterization of epitaxial semiconductor growth by reflectance anisotropy spectroscopy and ellipsometry, Prog. Cryst. Growth Charact. Mater. 35 (1997) 27-98.

[9] J. Olson, W.E. McMahon, Structure of Ge(100) surfaces for high-efficiency photovoltaic applications, in: Second World Conference and Exhibition on Photovoltaic Solar Energy Conversion, 1998.

[10] B. Galiana, K. Volz, 1. Rey-Stolle, W. Stolz, C. Algora, Influence of nucleation layers on MOVPE grown GaAs on Ge wafers for concentrator solar cells, in: Proceedings of the Fourth World Conference on Photovoltaic Energy Conversion, 2006, pp. 807-810.

[11] D.E. Aspnes, A.A. Studna, Anisotropies in the above-band-gap optical spectra of cubic semiconductors, Phys. Rev. Lett. 54 (1985) 1956-1959.

[12] T. Yasuda, I. Mantese, U. Rossow, D. Aspnes, Surface-induced optica anisotropies of single domain $(2 \times 1)$ reconstructed $(001) \mathrm{Si}$ and $(001) \mathrm{Ge}$ surfaces, Phys. Rev. Lett. 74 (1995) 3431-3434.

[13] H. Wormeester, D.J. Wentink, P.L. de Boeij, C.M.J. Wijers, A. van Silfhout, Surface states of the clean and oxidized $\mathrm{Ge}(001)$ surface studied with normal-incidence ellipsometry, Phys. Rev. B 47 (1993) 12663-12671. 
[14] S.M. Scholz, K. Jacobi, J.T. Zettler, W. Richter, Reflectance anisotropy spectroscopy on the Ge(113) surface, Surf. Sci. 359 (1996) 205-212.

[15] I. Horcas, R. Fernández, J.M. Gómez-Rodríguez, J. Colchero, J. Gómez-Herrero, A.M. Baro, WSXM: A software for scanning probe microscopy and a tool for nanotechnology, Rev. Sci. Instrum. 78 (2007) 013705.

[16] N.A. Kalyuzhnyy, V.M. Lantratov, S.A. Mintairov, M.A. Mintairov, M.Z. Shvarts, N.K. Timoshina, V. Andreev, In-situ monitoring during MOCVD growth of the triple-junction GalnP/Ga(In)As/Ge solar cells, in: Proceedings of the 23rd European Photovoltaic Solar Energy Conference, 2008.

[17] M. Zorn, M. Weyers, Application of reflectance anisotropy spectroscopy to laser diode growth in MOVPE, J. Cryst. Growth 276 (2005) 29-36.

[18] L. Lazzarini, L. Nasi, G. Salviati, C.Z. Fregonara, Y. Li, L.J. Giling, C. Hardingham, D.B. Holt, Antiphase disorder in GaAs/Ge heterostructures for solar cells, Micron 31 (2000) 217-222. 